\title{
I-band Cepheid Distance to NGC 6822
}

\author{
Myung Gyoon Lee ${ }^{1}$, Wendy L. Freedman ${ }^{1}$, Barry F. Madore ${ }^{2}$ \\ ${ }^{1}$ Carnegie Observatories, Pasadena, USA, ${ }^{2} I P A C / J P L / C a l t e c h$, Pasadena, USA
}

NGC 6822 was the first galaxy outside the Galaxy where the Cepheid periodluminosity relation was applied as a distance indicator (Hubble 1925, ApJ, 62, 409). Later, thirteen Cepheids in this galaxy were studied by Kayser (1967, AJ, 72, 134) using $B V$ photographic photometry. We have obtained BVRI CCD photometry of stars in two $2.2^{\prime} \times 3.5^{\prime}$ fields of NGC 6822. Field 3) using Figure 1 shows an $I-(V-I)$ diagram of $\sim 6,400$ measured stars in NGC 6822 including seven known Cepheids. The reddening is estimated to $E(B-V)=0.28 \pm 0.03$ from the $(B-$ $V)-(V-I)$ diagram of the stars with $\dot{V}<20$ mag. The distance to NGC 6822 has been estimated using the random-phase $I$ band photometry of seven Cepheids (V7(I=16.31), V5(I=18.97), V6(I=18.44), V3(I=17.88), V1( $I=18.05), \mathrm{V} 28(\mathrm{I}=17.61)$, and $\mathrm{V} 2(\mathrm{I}=17.29)$ ) combined with their periods given by Kayser. We have obtained $\Delta(m-M)_{I}(\mathrm{NGC} 6822-\mathrm{LMC})=5.48 \pm 0.17$ and $(m-M)_{0}=23.62 \pm 0.20$, adopting $(m-M)_{0}(\mathrm{LMC})=18.5$ and $A_{I}(\mathrm{NGC6822})=0.58$. The distance to NGC 6822 has also been estimated independently using the brightness of the tip of the red giant branch (RGB) of low mass stars following the method described in Da Costa \& Armandroff $(1990, \mathrm{AJ}, 100,162)$. The tip of the RGB is detected at $I=20.05 \pm 0.10$. This estimate yields a distance modulus of $(m-M)_{0}=23.46 \pm 0.20$, which is in good agreement with the Cepheid distance modulus. Taking the average of the Cepheid and TRGB distances, we obtain $\mathrm{f}$ WLM, $(m-M)_{0}=23.54 \pm 0.11$ corresponding to a distance of $510 \pm 30 \mathrm{kpc}$.

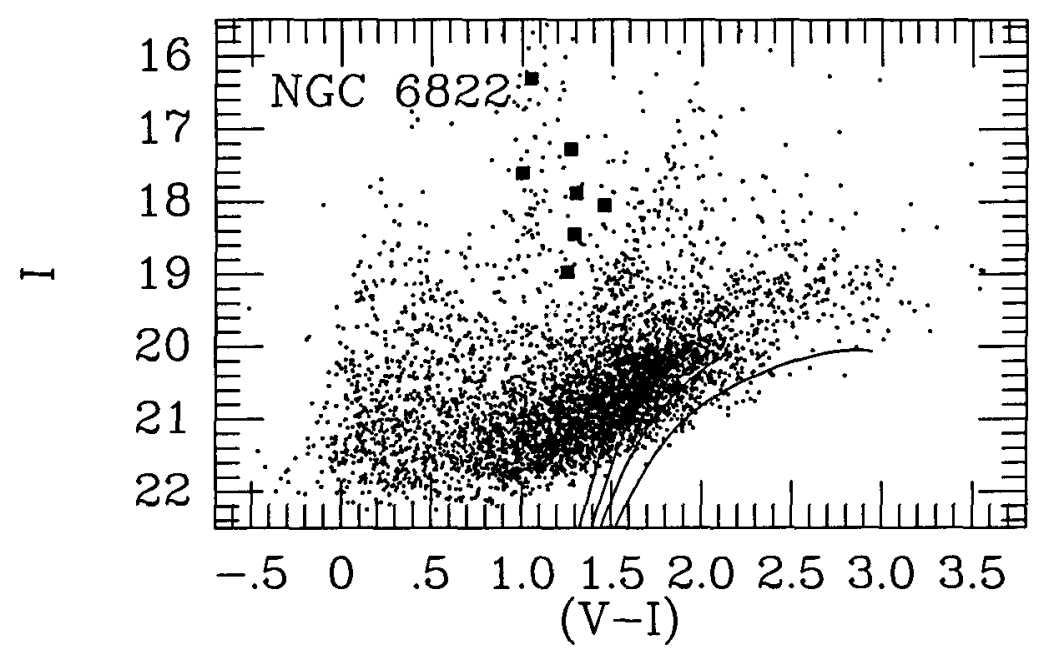

Fig. 1: $I-(V-I)$ diagram of $\sim 6,400$ measured stars in NGC 6822. The filled squares represent the Cepheids. The solid lines show the loci for the RGB of Galactic globular clusters, M15, M2, NGC 1851, and 47 Tuc (from Da Costa \& Armandroff). 